\title{
Metabolic syndrome impairs notch signaling and promotes apoptosis in chronically ischemic myocardium
}

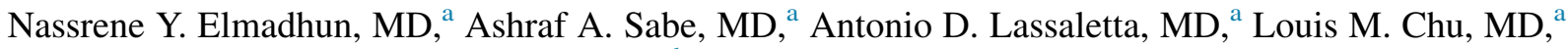 \\ Katelyn Kondra, BS, ${ }^{a}$ Michael Sturek, $\mathrm{PhD},{ }^{b}$ and Frank W. Sellke, $\mathrm{MD}^{\mathrm{a}}$
}

\begin{abstract}
Objective: Impaired angiogenesis is a known consequence of metabolic syndrome (MetS); however, the mechanism is not fully understood. Recent studies have shown that the notch signaling pathway is an integral component of cardiac angiogenesis. We tested, in a clinically relevant swine model, the effects of MetS on notch and apoptosis signaling in chronically ischemic myocardium.
\end{abstract}

\begin{abstract}
Methods: Ossabaw swine were fed either a regular diet (control [CTL], $\mathrm{n}=8$ ) or a high-cholesterol diet (MetS, $\mathrm{n}=8$ ) to induce MetS. An ameroid constrictor was placed to induce chronic myocardial ischemia. Eleven weeks later, the wine underwent cardiac harvest of the ischemic myocardium.

Results: Downregulation of pro-angiogenesis proteins notch2, notch4, jagged2, angiopoietin 1, and endothelial nitric oxide synthase were found in the MetS group compared with the CTL group. Also, upregulation of pro-apoptosis protein caspase 8 and downregulation of anti-angiogenesis protein phosphorylated forkhead box transcription factor 03 and pro-survival proteins phosphorylated P38 and heat shock protein 90 were present in the MetS group. Cell death was increased in the MetS group compared with the CTL group. Both CTL and MetS groups had a similar arteriolar count and capillary density, and notch 3 and jagged1 were both similarly concentrated in the smooth muscle wall.

Conclusions: MetS in chronic myocardial ischemia significantly impairs notch signaling by downregulating notch receptors, ligands, and pro-angiogenesis proteins. MetS also increases apoptosis signaling, decreases survival signaling, and increases cell death in chronically ischemic myocardium. Although short-term angiogenesis appears unaffected in this model of early MetS, the molecular signals for angiogenesis are impaired, suggesting that inhibition of notch signaling might underlie the decreased angiogenesis in later stages of MetS. (J Thorac Cardiovasc Surg 2014;148:1048-55)
\end{abstract}

The metabolic syndrome (MetS) is a cluster of metabolic derangements that includes obesity, insulin resistance, glucose intolerance, dyslipidemia, and hypertension. MetS substantially increases the risk of cardiovascular disease and mortality. ${ }^{1,2}$ Despite advancements in surgical technique and percutaneous interventions, patients with hypertension, hyperlipidemia, and diabetes have had significantly greater mortality after angioplasty, coronary stenting, and coronary artery bypass grafting. ${ }^{3}$ The

From the Division of Cardiothoracic Surgery, ${ }^{\mathrm{a}}$ Cardiovascular Research Center, Brown University Warren Alpert School of Medicine, Providence, RI; and Department of Cellular and Integrative Physiology, ${ }^{\mathrm{b}}$ Indiana University School of Medicine, Indianapolis, Ind.

Disclosures: Frank W. Sellke reports consulting fees from CSL Behring, the Medicines Company, and Pfizer. All other authors have nothing to disclose with regard to commercial support.

Read at the 94th Annual Meeting of The American Association for Thoracic Surgery, Toronto, Ontario, Canada, April 26-30, 2014.

Received for publication April 9, 2014; revisions received May 16, 2014; accepted for publication May 21, 2014; available ahead of print July 16, 2014.

Address for reprints: Frank W. Sellke, MD, Division of Cardiothoracic Surgery, Cardiovascular Research Center, Brown University Warren Alpert School of Medicine, 2 Dudley St, MOC 360, Providence, RI 02905 (E-mail: fsellke@ lifespan.org).

$0022-5223 / \$ 36.00$

Copyright (c) 2014 by The American Association for Thoracic Surgery

http://dx.doi.org/10.1016/j.jtcvs.2014.05.056 promising results from animal studies in therapeutic revascularization with growth factor, gene, or cell therapy have met with disappointing results in humans, with minimal clinical improvements in myocardial angiogenesis. ${ }^{4}$ Not surprisingly, young healthy animals with chronic myocardial ischemia are better able to adapt to the ischemic insult, but older patients with multiple comorbidities, including MetS, have limited myocardial adaptability to ischemia. The discordant results between positive findings from animal studies and negative clinical results highlights the need to understand the effects of MetS on the molecular angiogenic signaling pathways using a clinically relevant animal model.

Ossabaw miniswine are a breed of feral pigs that were isolated on Ossabaw Island off the coast of Georgia almost 500 years ago by Spanish explorers. This pig breed has been identified as an excellent model for MetS owing to its "thrifty genotype," which allowed these pigs to adapt to the harsh island conditions by storing large amounts of fat during the feasting period. ${ }^{5}$ When sedentary and fed a high-fat, high-calorie, atherogenic diet, these swine develop profound obesity and all the hallmark features of MetS and are, thus, a useful model to study MetS and coronary artery disease..$^{5-7}$

We recently demonstrated that animals with chronic myocardial ischemia and perivascular vascular endothelial growth factor (VEGF) had significantly improved 


\section{Abbreviations and Acronyms \\ Ang1 = angiopoietin 1 \\ DLL $=$ delta like ligand \\ eNOS = endothelial nitric oxide synthase \\ MetS = metabolic syndrome \\ $\mathrm{NIH}=$ National Institutes of Health \\ SMA $=$ smooth muscle actin \\ TUNEL $=$ terminal deoxynucleotidyl transferase- mediated deoxyuridine triphosphate-biotin nick end labeling \\ VEGF = vascular endothelial growth factor \\ VEGFR $=$ VEGF receptor}

neovascularization and Notch receptor and ligand expression. ${ }^{8}$ The Notch signaling pathway is an evolutionarily conserved pathway important for many processes, including cell fate determination differentiation, proliferation, apoptosis, and regeneration. ${ }^{9}$ Studies have shown that postnatal Notch signaling is critical for angiogenesis. ${ }^{10,11}$ Interest is growing in the clinical utility of Notch modulators to suppress angiogenesis in tumors and promote angiogenesis in ischemic myocardium. ${ }^{12}$ Although research in the role of Notch signaling in developmental biology is longstanding, the role of Notch signaling in mature myocardium in response to ischemia and MetS is largely unknown. The purpose of the present study was to examine the effects of MetS on Notch signaling in response to chronic myocardial ischemia in the clinically relevant Ossabaw model of early MetS.

\section{METHODS}

\section{Animal Model}

Sixteen intact male Ossabaw miniswine (Purdue Ossabaw Facility, Indiana University, Indianapolis, Ind) were split into 2 groups according to diet at 6 weeks of age. The control (CTL) group was fed $500 \mathrm{~g} / \mathrm{d}$ of regular chow $(\mathrm{n}=8)$. The high-cholesterol swine were fed $500 \mathrm{~g} / \mathrm{d}$ of high-cholesterol chow (MetS group, $\mathrm{n}=8$ ) consisting (by weight) of $4 \%$ cholesterol, $17.2 \%$ coconut oil, $2.3 \%$ corn oil, $1.5 \%$ sodium cholate, and 75\% regular chow (Sinclair Research, Columbia, Mo). After 9 weeks of diet initiation, all the swine underwent surgical placement of an ameroid constrictor to induce chronic myocardial ischemia (see "Surgical Interventions"). Postoperatively, all the swine continued their respective diets. Eleven weeks after ameroid constrictor placement, all the swine underwent euthanasia and cardiac tissue harvest. All the swine were observed to ensure complete consumption of food and unlimited access to water and were housed in a warm nonstressful environment for the duration of the experiment.

\section{Surgical Interventions}

Anesthesia. Anesthesia was induced with an intramuscular injection of telazol $(4.4 \mathrm{mg} / \mathrm{kg})$. The swine were endotracheally intubated and mechanically ventilated at 12 to 20 breaths $/ \mathrm{min}$. General anesthesia was maintained with a gas mixture of oxygen at 1.5 to $2 \mathrm{~L} / \mathrm{min}$ and $0.75 \%$ to $3.0 \%$ isoflurane.
Ameroid constrictor placement. For ameroid constrictor placement, the swine were given a single dose of intravenous enrofloxacin $(5 \mathrm{mg} / \mathrm{kg}$ ) for antibiotic prophylaxis, and general anesthesia was induced and maintained. The swine were prepared and draped in the usual sterile fashion. The heart was exposed through a left minithoracotomy through the fourth intercostal space and pericardiotomy. The left atrial appendage was retracted, and the left circumflex artery was dissected at the take off of the left main coronary artery. A titanium ameroid constrictor (1.75-2.25 mm internal diameter) ameroid constrictor was placed around the proximal left circumflex artery, just after its take off from the left main coronary artery (Research Instruments SW, Escondito, Calif). The pericardium was loosely reapproximated with interrupted 4-0 Nurolon sutures (Ethicon, Somerville, NJ), followed by a layered closure of the surgical incision. Postoperative pain was controlled by a single dose of intramuscular buprenorphine $(0.03 \mathrm{mg} / \mathrm{kg})$ and a 72 -hour fentanyl patch (4 $\mu \mathrm{g} / \mathrm{kg}$ ). All the swine received $325 \mathrm{mg}$ of aspirin daily starting 1 day preoperatively and continuing for 5 days for prophylaxis against thromboembolic events. All the swine continued perioperative antibiotics (enrofloxacin $68 \mathrm{mg}$ orally daily for 5 days).

Cardiac harvest. For cardiac harvest, with the swine under general anesthesia, the heart was exposed by way of a median sternotomy, and the swine were euthanized by exsanguination. Cardiac tissue from the ischemic territory in the left circumflex artery distribution was collected for additional analysis. The institutional animal care and use committee of the Rhode Island Hospital approved all experiments. The swine were cared for in compliance with the "Principles of Laboratory Animal Care" formulated by the National Society for Medical Research and the "Guide for the Care and Use of Laboratory Animals" (National Institutes of Health [NIH] publication no. 5377-3, 1996).

\section{Immunohistochemical Staining for Angiogenesis}

For immunohistochemical staining for angiogenesis, frozen myocardium was sectioned (10- $\mu \mathrm{m}$ thickness) and fixed in $10 \%$ formalin for 10 minutes. The sections were blocked with $1 \%$ bovine serum albumin in phosphate-buffered saline for 1 hour at room temperature and incubated with antibodies against the porcine endothelial marker CD31 (R\&D Systems, Minneapolis, Minn) and smooth muscle actin (SMA) (Sigma-Aldrich, St Louis, Mo), followed by the appropriate Alexa-Fluor conjugated antibody (Jackson ImmunoResearch, West Grove, Pa) for 45 minutes. Slides were then mounted with $46^{\prime}$-diamidino-2-phenylindole-2 $\mathrm{HCl}$-containing medium (Vectashield; Vector Laboratories, Burlingame, Calif). Images were captured at $\times 20$ magnification with a Nikon E800 Eclipse microscope (Nikon, Tokyo, Japan) at the same exposure in 3 random fields. The capillaries were defined as structures 5 to $25 \mu \mathrm{m}^{2}$ in cross-sectional area, and arterioles were defined by co-localization of SMA (red) and CD31 (green) staining. The arteriolar and capillary density were measured using Image $\mathrm{J}$ software $(\mathrm{NIH}$, Bethesda, Md). The percentage of capillary and arteriolar density for each pig was averaged from the 3 randomly selected myocardial tissue sections.

\section{Protein Expression}

To determine protein expression, $40 \mu \mathrm{g}$ of the radioimmunoprecipitation assay (Boston BioProducts, Ashland, Mass) soluble fraction of myocardial lysates was fractionated using sodium dodecyl sulfate polyacrylamide gel electrophoresis $3 \%$ to $8 \%$ Tris-acetate gel (NuPage Novex Mini Gel; Invitrogen, Carlsbad, Calif) for molecular weight targets $>100 \mathrm{kDa}$ and $4 \%$ to $12 \%$ Bis-Tris gels for molecular weight targets $<100 \mathrm{kDa}$ (NuPage Novex Mini Gel; Invitrogen). The protein was then transferred to polyvinylidene difluoride membranes (Millipore, Billerica, Mass) and incubated overnight at $4^{\circ} \mathrm{C}$ with primary antibodies at the dilutions recommended by the manufacturer against Notch1, Notch2, Notch3, Notch4, Jagged1, Jagged2, VEGF receptor (VEGFR)2, vascular endothelial cadherin, endothelial nitric oxide synthase (eNOS), 
transforming growth factor- $\beta$, caspase 8 , caspase 3, cleaved caspase 3, forkhead box transcription factor 03 (Fox03), phosphorylated Fox03, apoptosis inducing factor, P38, phosphorylated P38, heat shock protein 90 (all from Cell Signaling, Danvers, Mass) and VEGFR3 and angiopoietin 1 (Ang1; both from Abcam, Cambridge, Mass). The membranes were incubated with the appropriate horseradish peroxidase-linked secondary antibody for 1 hour at room temperature (Jackson ImmunoResearch, West Grove, Pa). Immune complexes were visualized with enhanced chemiluminescense, and the images were captured with a digital camera system (G-Box; Syngene, Cambridge, United Kingdom). Band densitometry was quantified as arbitrary light units using Image-J software (NIH). All membranes were probed with glyceraldehyde-3-phosphate dehydrogenase (Cell Signaling) to correct for loading error.

\section{Immunohistochemical Staining for Notch Signaling Proteins}

For immunohistochemical staining for Notch signaling proteins, frozen myocardium was sectioned (10- $\mu$ m thickness) and fixed in $10 \%$ formalin for 10 minutes. The sections were blocked with $1 \%$ bovine serum albumin in phosphate-buffered saline for 1 hour at room temperature and incubated with antibodies against Notch2, Notch3, Notch4, or Jagged1 (Cell Signaling) and SMA (Sigma-Aldrich) co-staining, followed by the appropriate Alexa-Fluor conjugated antibody (Jackson ImmunoResearch) for 45 minutes. The slides were then mounted with Vectashield (Vector Laboratories). The images were captured at $\times 20$ magnification with a Nikon E800 Eclipse microscope (Nikon) at the same exposure in 3 random fields. The immunofluorescence mean intensity was measured using ImageJ software (NIH). The immunofluorescence mean intensity for each pig was averaged from the 3 randomly selected myocardial tissue sections.

\section{Terminal Deoxynucleotidyl Transferase-Mediated Deoxyuridine Triphosphate-Biotin Nick End \\ Labeling Staining}

For terminal deoxynucleotidyl transferase-mediated deoxyuridine triphosphate-biotin nick end labeling (TUNEL) staining, frozen myocardium from the ischemic territory was sectioned (10- $\mu \mathrm{m}$ thickness). Apoptotic cells were identified using the commercially available ApopTag detection kit (Millipore), according to the manufacturer's instructions. The images were captured at $\times 20$ magnification using Aperio ScanScope technology (Leica Biosystems, Vista, Calif) in 3 random fields from each pig. The percentage of TUNEL-positive cardiomyocytes per high powered field was measured using ImageJ software (NIH). The percentage of cell death per high powered field for each pig was averaged from the 3 randomly selected myocardial tissue sections.

\section{Statistical Analysis}

All results are reported as the mean \pm standard error of the mean. Student's $t$ test was used to compare the mean values using GraphPad Prism, version 5.0, Software (GraphPad Software, Inc, San Diego, Calif). Protein expression is reported as the fold change compared with the CTL group.

\section{RESULTS \\ Animal Model}

All the swine included in the present analysis survived the entire experiment. One pig in the CTL group died on postoperative day 8 , and 1 in the MetS group died on postoperative day 2. The necropsy findings were unremarkable, and we assumed that both pigs had died of an acute ventricular arrhythmia. The pigs that did not survive to completion of the experiment were excluded from analysis and replaced with new animals. We have previously reported that no difference was seen in myocardial perfusion or contractility. ${ }^{13} \mathrm{We}$ also reported that the pigs in the study group developed key components of MetS, including obesity (body mass index 31.9 vs $35.1 \mathrm{~kg} / \mathrm{m}^{2}$ for CTL vs MetS, respectively; $P<.01$ ), hypertension (mean arterial pressure, 85 vs $112 \mathrm{~mm} \mathrm{Hg}$ for CTL vs MetS, respectively; $P=.02$ ), dyslipidemia (67.9 vs 343 $\mathrm{mg} / \mathrm{dL}$ total cholesterol for CTL vs MetS, respectively; $P<.01$ ), and glucose intolerance in response to dextrose infusion $(44,149$, and $82 \mathrm{mg} / \mathrm{dL}$ blood glucose in the CTL group vs 60, 214, and $130 \mathrm{mg} / \mathrm{dL}$ in the MetS group at 0,30 , and 60 minutes, respectively, after dextrose infusion; $P<.01){ }^{13}$

\section{Vessel Density in Chronically Ischemic Myocardium}

No difference was seen in the capillary density in the chronically ischemic myocardium as measured by immunohistochemical staining for CD31 $(9.53 \% \pm 0.72 \%$ and $10.78 \% \pm 0.74 \%$ area/high power field for CTL and MetS, respectively; $P=.28$ ). Also, no difference was seen in the arteriolar count as measured by immunohistochemical co-staining of CD31 and SMA (22.91 \pm 1.18 and $23.73 \pm 0.93$ count/high power field for CTL and MetS, respectively; $P=.59$; Figure 1).

\section{Angiogenesis Protein Expression in Chronically Ischemic Myocardium}

We analyzed the myocardial lysates of the chronically ischemic myocardium for expression of all 4 mammalian Notch receptors (Notch1-4) and 2 Notch ligands (Jagged1 and 2). In the MetS group, a decrease was seen in the expression of Notch2, Notch4, and Jagged2 compared with that in the CTL group. Also, decreased expression of pro-angiogenesis proteins Ang1 and eNOS was seen in the MetS group compared with the CTL group. No difference was found in the expression of Notch1, Notch3, Jagged 1, VEGFR1, VEGFR2, and transforming growth factor $-\beta$ in the CTL and MetS groups (Table 1).

\section{Notch Receptor and Ligand Staining in Chronically Ischemic Myocardium}

We performed immunohistochemical staining of transmural sections of the ischemic myocardium to determine the histologic location of the Notch2, Notch3, and Notch4 receptors by co-staining with SMA. SMA stains vascular smooth muscle, which is located in the wall of the arteries and arterioles. Notch receptors Notch2, Notch3, and Notch4 and Notch ligand Jagged1 (green) were highly concentrated in the smooth muscle wall and co-localized with SMA (red; Figures 2 and 3). Although no difference was seen in overall Notch3 intensity in the CTL and MetS groups, a significant decrease was seen in Notch2 and 

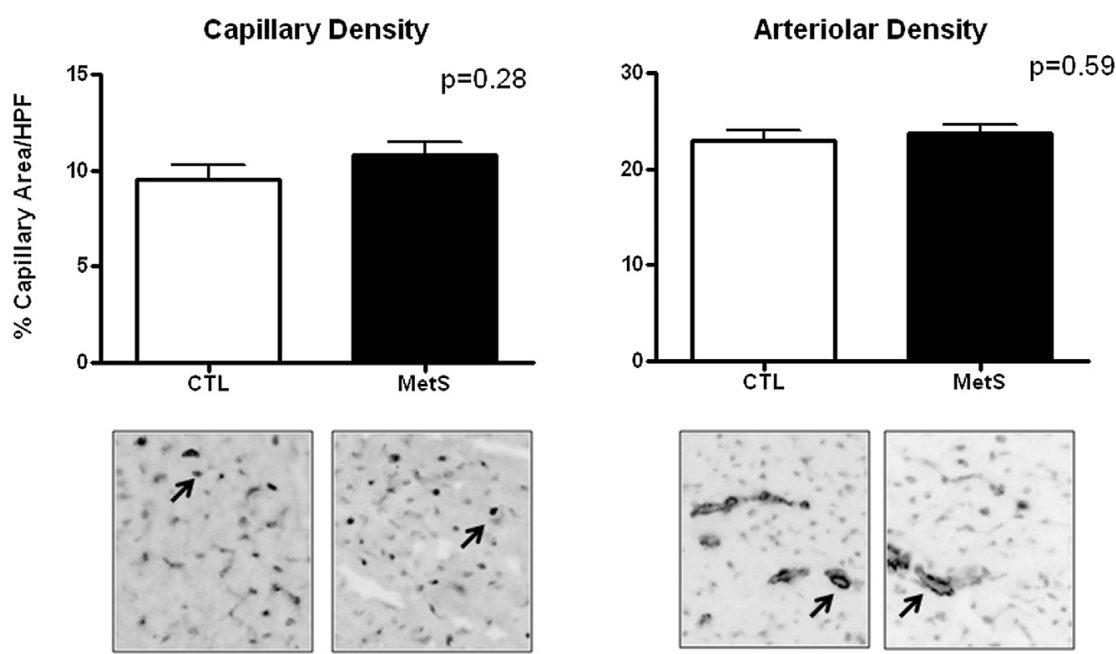

FIGURE 1. Vessel density in chronically ischemic myocardium. Left, Arrows indicate capillaries; right, arrows indicate arterioles. CTL, Ossabaw control group; MetS, Ossabaw atherogenic diet (to induce metabolic syndrome) group; HPF, high-powered field.

Notch4 intensity in the MetS group compared with the CTL group. A trend was seen toward decreased Jagged1 immunofluorescence in the MetS group compared with the CTL group, but the difference did not reach statistical significance.

\section{Apoptosis and Survival Protein Expression in Chronically Ischemic Myocardium}

We analyzed the myocardial lysates of the chronically ischemic myocardium for the expression of apoptosis and survival signaling proteins. We found that in the MetS group, upregulation of pro-apoptosis protein caspase 8 and downregulation of the inhibited form of pro-apoptosis protein Fox03, phosphorylated Fox03, were present compared with the CTL group. Also, significant downregulation of

TABLE 1. Angiogenesis protein expression in chronically ischemic myocardium

\begin{tabular}{lccc}
\hline Variable & CTL group & MetS group & $\boldsymbol{P}$ value \\
\hline Notch1 & $1.0 \pm 0.132$ & $0.81 \pm 0.053$ & .20 \\
Notch2 & $1.0 \pm 0.105$ & $0.64 \pm 0.096$ & .03 \\
Notch3 & $1.0 \pm 0.076$ & $1.11 \pm 0.088$ & .38 \\
Notch4 & $1.0 \pm 0.128$ & $0.66 \pm 0.050$ & .02 \\
Jagged1 & $1.0 \pm 0.143$ & $1.17 \pm 0.151$ & .43 \\
Jagged2 & $1.0 \pm 0.050$ & $0.79 \pm 0.083$ & .05 \\
VEGFR2 & $1.0 \pm 0.082$ & $1.12 \pm 0.127$ & .43 \\
VEGFR3 & $1.0 \pm 0.100$ & $1.05 \pm 0.107$ & .76 \\
VE cadherin & $1.0 \pm 0.052$ & $0.82 \pm 0.113$ & .18 \\
Ang1 & $1.0 \pm 0.070$ & $0.72 \pm 0.045$ & .01 \\
eNOS & $1.0 \pm 0.038$ & $0.51 \pm 0.126$ & .003 \\
TGF- $\beta$ & $1.0 \pm 0.037$ & $0.98 \pm 0.070$ & .77 \\
\hline D &
\end{tabular}

Data presented as fold change \pm standard error of the mean compared with the CTL group. CTL, Control; MetS, Ossabaw atherogenic diet (to induce metabolic syndrome); VEGFR, vascular endothelial growth factor receptor; $V E$, vascular endothelial; Angl, angiopoietin 1; eNOS, endothelial nitric oxide synthase; $T G F$, transforming growth factor. the pro-survival proteins phosphorylated P38 and heat shock protein 90 was present in the MetS group compared with the CTL group. Also, increased expression of pro-apoptosis proteins cleaved caspase 3 and apoptosis inducing factor was seen in the MetS group, but the difference did not reach statistical significance $(P=.06$ and $P=.09$, respectively) compared with the CTL group (Table 2). No difference was found in the expression of pro-apoptosis proteins caspase 3 and Fox03 or pro-survival protein P38.

\section{TUNEL Staining in Chronically Ischemic Myocardium}

We sampled the chronically ischemic myocardium and performed TUNEL staining to quantify the cell death in the CTL and MetS groups. We found a significant increase in cardiomyocyte cell death in the MetS group compared with the CTL group $(2.73 \pm 0.36$ vs $1.71 \pm 0.34$, respectively; $P=.05$; Figure 4 ).

\section{DISCUSSION}

Coronary collateralization in response to chronic myocardial ischemia is an important endogenous mechanism to preserve myocardial function, limit infarct size, and improve long-term survival. It is therefore critical to understand the molecular basis for how the MetS inhibits coronary collateralization and angiogenesis. In this clinically relevant swine model of chronic myocardial ischemia, we found that early MetS attenuated Notch, angiogenesis, and survival signaling and upregulated apoptosis signaling in chronically ischemic myocardium. Previously, we had demonstrated that feeding Ossabaw swine a high-fat, high-calorie, atherogenic diet resulted in all the hallmark components of MetS, including obesity, insulin resistance, glucose intolerance, elevated blood pressure, and hyperlipidemia. ${ }^{13,14}$ 

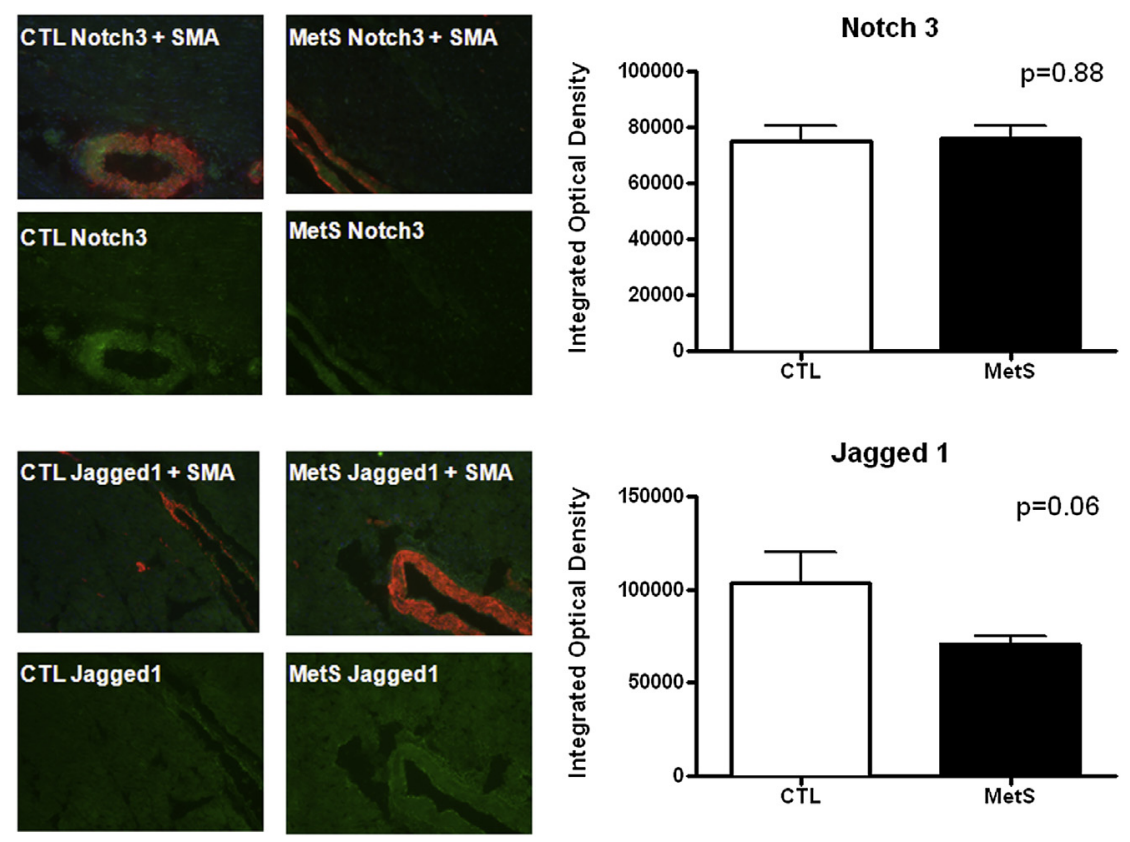

FIGURE 2. Notch receptor and ligand staining in chronically ischemic myocardium. Top, Notch3 (green) and smooth muscle actin (SMA; red). Bottom, Notch ligand jagged1 (green). CTL, Ossabaw control group; MetS, Ossabaw atherogenic diet (to induce metabolic syndrome) group.

The Notch signaling pathway is a short-range communication system between 2 physically adjacent cells, so-called juxtacrine signaling. Mammals have 4 Notch receptors, Notch 1 to 4 , and 5 ligand receptors, delta like ligand (DLL)1, 3, and 4 and Jagged1 and 2. The vascular endothelium only expresses the receptors Notch1, Notch2, and Notch4 and the ligands Jagged1, Jagged2, DLL1, and DLL4. ${ }^{12}$ Notch3 is predominantly expressed in the adult arterial smooth muscle wall. ${ }^{15}$ Notch signaling is an integral part of both angiogenesis and vasculogenesis and is required for arterial and venous blood vessel differentiation. ${ }^{16}$ The Notch pathway also maintains endothelial
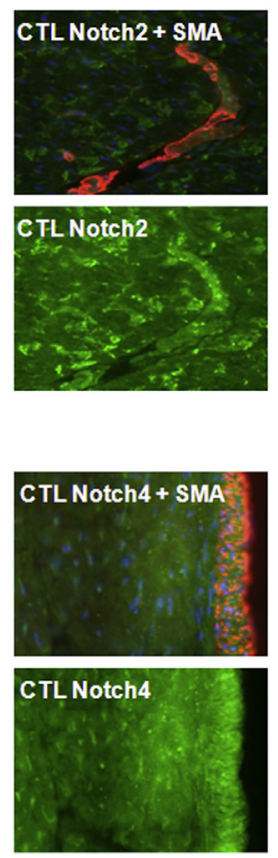
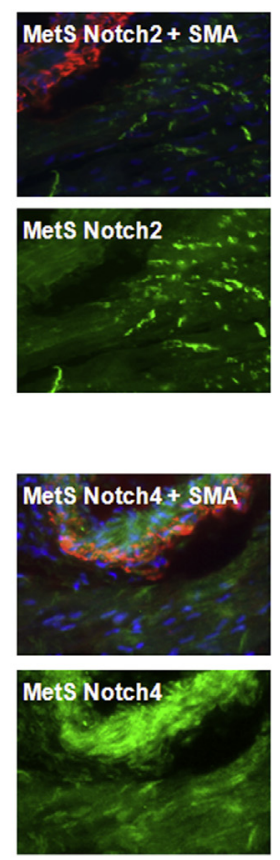

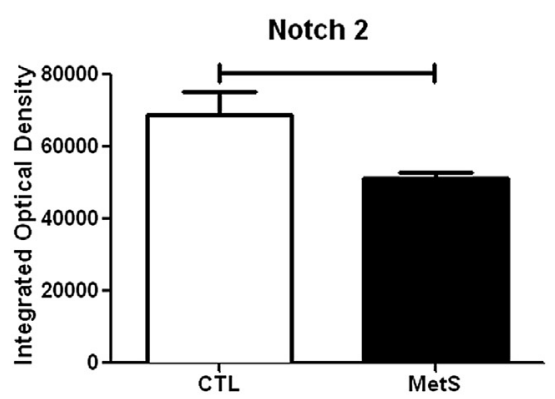

Notch 4

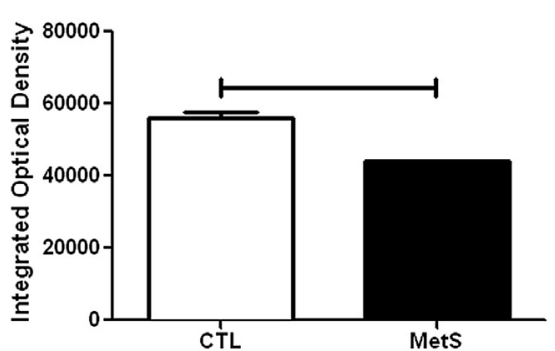

FIGURE 3. Notch receptor staining in chronically ischemic myocardium. Top, Notch2 (green); bottom, Notch4 (green). Red indicates smooth muscle actin (SMA). CTL, Ossabaw control group; MetS, Ossabaw atherogenic diet (to induce metabolic syndrome) group. 
TABLE 2. Apoptosis and survival protein expression in chronically ischemic myocardium

\begin{tabular}{lccc}
\hline \multicolumn{1}{c}{ Variable } & CTL & MetS & $\boldsymbol{P}$ value \\
\hline Caspase 8 & $1.0 \pm 0.051$ & $1.19 \pm 0.051$ & .02 \\
Caspase 3 & $1.0 \pm 0.065$ & $1.04 \pm 0.093$ & .72 \\
Cleaved caspase 3 & $1.0 \pm 0.162$ & $1.55 \pm 0.210$ & .06 \\
Fox03 & $1.0 \pm 0.025$ & $0.95 \pm 0.033$ & .27 \\
pFox03 (Ser253) & $1.0 \pm 0.039$ & $0.84 \pm 0.040$ & .01 \\
AIF & $1.0 \pm 0.108$ & $1.32 \pm 0.136$ & .09 \\
P38 & $1.0 \pm 0.028$ & $1.03 \pm 0.053$ & .61 \\
pP38 (Thr180/Tyr182) & $1.0 \pm 0.052$ & $0.80 \pm 0.029$ & .01 \\
HSP90 & $1.0 \pm 0.038$ & $0.85 \pm 0.052$ & .04 \\
\hline
\end{tabular}

Data presented as fold change \pm standard error of the mean compared with the CTL group. CTL, Control; MetS, Ossabaw atherogenic diet (to induce metabolic syndrome); Fox03, forkhead box transcription factor 03; pFox03, phosphorylated Fox $03 ; A I F$, apoptosis inducing factor; $p P 38$, phosphorylated $\mathrm{P} 39 ; H S P 90$, heat shock protein 90 .

homeostasis by modulating new blood vessel sprouting by way of DLL4/Notch1; protecting endothelial cells from tumor necrosis factor- $\alpha$-mediated apoptosis by way of Notch4; and regulating bone marrow endothelial progenitor cell proliferation, apoptosis, and migration to ischemic tissue by way of Notch $1 .{ }^{12}$

The Notch pathway has been proposed as a possible target for therapeutic angiogenesis and cardioprotection; however, studies in a large animal model with clinically relevant comorbidities, such as MetS, are lacking. ${ }^{12}$ In the present study, we have demonstrated that MetS has an inhibitory effect on Notch signaling. We found that Notch receptors Notch2, Notch3, and Notch4 and Notch ligand Jagged 1 were primarily concentrated in the vascular smooth muscle wall and co-localized with SMA. This was an expected result given that Notch3 is expressed in vascular smooth muscle cells. We also found decreased Notch2 and Notch2 staining and a trend toward decreased Jagged1 tissue staining in the MetS group compared with the CTL group, suggesting that decreased Notch signaling occurs in the vascular smooth muscle wall in animals with MetS. Jagged 1 has been identified as an important Notch target during arterial wall assembly, with Jagged1 inhibition resulting in arterial defects and impaired vascular smooth muscle differentiation. ${ }^{17}$ Our results suggest that perhaps MetS inhibits the Notch signaling angiogenic response to chronic myocardial ischemia. We have also reported that downregulation occurred of pro-angiogenic proteins eNOS and Ang1. Previous studies have demonstrated a direct link between Notch signaling and eNOS activity. ${ }^{18,19}$ Notch activation activates eNOS, which induces nitric oxide synthesis. In an animal model of hind limb ischemia, Notch inhibition in endothelial cells reduced reperfusion and nitric oxide generation. ${ }^{18}$ Ang1 is an angiogenic factor that also activates the pro-survival signaling pathway and initiates stem cell homing in the setting of myocardial ischemia. ${ }^{20}$ In a porcine model of myocardial infarction, co-expression of VEGF and Ang1 was shown to increase angiogenesis, myocardial perfusion, and cardiomyocyte proliferation in ischemic myocardium. ${ }^{21}$ In the present study, MetS had an inhibitory effect on the normal angiogenic response to ischemia by downregulating eNOS and Ang1.

It was somewhat surprising that in this model of early MetS in Ossabaw swine, we did not observe a reduction in collateral-dependent myocardial perfusion, which we have reported using other strains of pigs. ${ }^{22,23}$ This might be specific to the Ossabaw pigs and in part a result of increased vascular relaxation observed using these pigs in early-stage MetS. ${ }^{13}$ Perhaps the increased microvascular relaxation results from upregulation of the peroxisome proliferator-activated receptor pathway. It could also be related to the "thrifty genotype," which enabled this pig breed to survive in the feast and famine ecology on Ossabaw Island, despite their predilection to obesity and coronary artery disease. It could also be that this transient improvement in microvessel reactivity we had previously reported also occurs in humans in the presence of early MetS. Later stage MetS in Ossabaw swine, in contrast, has been associated with decreased endotheliumdependent relaxation and coronary flow reserve, indicating the progression in MetS severity. ${ }^{6,24-26}$ Given the decreased expression of Notch and angiogenesis signaling proteins, one would expect there would also be decreased vessel density. In the present study, however, we did not find any

TUNEL Stain

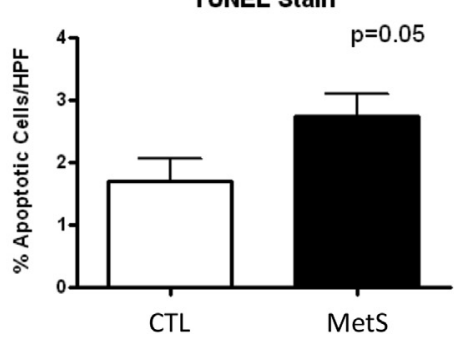

FIGURE 4. Terminal deoxynucleotidyl transferase-mediated deoxyuridine triphosphate-biotin nick end labeling (TUNEL) staining in chronically ischemic myocardium. Brown spots (arrow) in nuclei indicate cell death. CTL, Control group; MetS, Ossabaw atherogenic diet (to induce metabolic syndrome) group; $H P F$, high-powered field. 
difference in arteriolar or capillary density in the MetS group compared with the CTL group. This finding might have been because these pigs had been supplemented with a high-fat diet for a relatively short period and developed early-stage MetS. Importantly, the molecular changes in angiogenesis protein expression precede the histologic changes and reductions in vessel count. If the pigs were fed the high-fat diet for a longer period and had longstanding chronic MetS, the molecular downregulation of Notch and angiogenesis signaling would manifest in a histologically visible reduction in angiogenesis. Trask and colleagues $^{24}$ reported decreased capillary density in Ossabaw pigs that were older and had had MetS for a longer duration.

In addition to its pro-angiogenic properties, Notch signaling has been found to modulate apoptosis and survival signaling. In cell cultures, pro-apoptotic tumor necrosis factor- $\alpha$ administration downregulated Notch 4 activity. ${ }^{27}$ Notch 4 also increased expression of Bcl2, thereby protecting endothelial cells from lipopolysaccharide-triggered apoptosis. ${ }^{28}$ Notch1 signaling in rat cardiomyocytes reduced apoptosis in ischemic preconditioning by upregulating Bcl2 and downregulating Bax. ${ }^{29}$ Thus, Notch signaling could be used to promote endothelial generation and angiogenesis and inhibit endothelial and cardiomyocyte apoptosis. Studies in oncologic biology have shown that Notch signaling inhibits apoptosis through the Akt pathway in neoplastic tissues. ${ }^{30,31}$ In the present study, we examined the effect of the MetS on apoptosis-survival signaling in ischemic myocardium. A significant decrease was seen in the pro-survival proteins phosphorylated P38 and heat shock protein 90, a decrease in the inhibited form of pro-apoptosis protein phosphorylated Fox03, and upregulation of pro-apoptosis protein caspase 8 in the MetS group. We also saw a trend toward increased expression of the pro-apoptosis proteins apoptosis inducing factor and cleaved caspase 3 in the MetS group. The increase in pro-apoptosis protein expression and decrease in the expression of survival proteins in the MetS group was supported by TUNEL staining, which demonstrated increased cell death in the MetS group compared with the CTL group. These results suggest that MetS promotes apoptosis signaling and increases cell death in the setting of chronic myocardial ischemia. Although we have previously reported that no difference was present in cardiac function, long-term MetS-mediated cell death might result in decreased contractility and reduced adaptability to myocardial ischemia. ${ }^{8}$ Our finding of decreased Notch signaling in this early-stage MetS model is consistent with these molecular signals being causally related to decreased angiogenesis in later stage MetS. ${ }^{24}$

The MetS poses a significant public health dilemma, and its prevalence is increasing, with no signs of slowing. ${ }^{32}$ Notch signaling has been established as an integral component of angiogenesis and cell survival in the postnatal heart. Elucidating this complex signaling network could assist in identifying possible Notch targets and its applicability and efficacy in human pathologic entities. We have provided evidence that MetS inhibits Notch signaling and promotes cell death in the chronically ischemic myocardium, which could be one way that MetS negatively affects overall cardiac health and increases the risk of developing cardiovascular disease.

\section{Study Limitations}

The present study had several limitations in the presentation and analysis. Although the swine in our study had early MetS, the known vascular consequences of MetS were not demonstrated in the chronically ischemic myocardium (ie, no difference was seen in vessel count or microvessel relaxation). In future studies, it would be helpful to repeat the study with multiple harvest points to establish a time course for the molecular and histologic changes in angiogenesis in the setting of ischemia and early and established MetS. Furthermore, although we found differences in Notch expression in the present animal model of early MetS and chronic ischemia, causality with angiogenesis has not been clearly established. Future studies with directed upregulation of Notch receptor or ligands are warranted to further elucidate the relationship among Notch signaling, MetS, and chronic myocardial ischemia.

\section{References}

1. Malik S, Wong ND, Franklin SS, Kamath TV, L'Italien GJ, Pio JR, et al. Impact of the metabolic syndrome on mortality from coronary heart disease, cardiovascular disease, and all causes in united states adults. Circulation. 2004; 110:1245-50.

2. Mensah GA, Mokdad AH, Ford E, Narayan KM, Giles WH, Vinicor F, et al. Obesity, metabolic syndrome, and type 2 diabetes: emerging epidemics and their cardiovascular implications. Cardiol Clin. 2004;22:485-504.

3. Volzke H, Henzler J, Menzel D, Robinson DM, Hoffmann W, Vogelgesang D, et al. Outcome after coronary artery bypass graft surgery, coronary angioplasty and stenting. Int J Cardiol. 2007;116:46-52.

4. Lassaletta AD, Chu LM, Sellke FW. Therapeutic neovascularization for coronary disease: current state and future prospects. Basic Res Cardiol. 2011;106: 897-909.

5. Dyson MC, Alloosh M, Vuchetich JP, Mokelke EA, Sturek M. Components of metabolic syndrome and coronary artery disease in female Ossabaw swine fed excess atherogenic diet. Comp Med. 2006;56:35-45.

6. Neeb ZP, Edwards JM, Alloosh M, Long X, Mokelke EA, Sturek M. Metabolic syndrome and coronary artery disease in Ossabaw compared with Yucatan swine. Comp Med. 2010;60:300-15.

7. Lee L, Alloosh M, Saxena R, Van Alstine W, Watkins BA, Klaunig JE, et al. Nutritional model of steatohepatitis and metabolic syndrome in the Ossabaw miniature swine. Hepatology. 2009;50:56-67.

8. Lassaletta AD, Elmadhun NY, Burgess TA, Bianchi C, Sabe AA, Robich MP, et al. Microvascular notch signaling is upregulated in response to vascular endothelial growth factor and chronic myocardial ischemia. Circ J. 2014;78: 743-51.

9. Fortini ME. Notch signaling: the core pathway and its posttranslational regulation. Dev Cell. 2009;16:633-47.

10. Chang L, Noseda M, Higginson M, Ly M, Patenaude A, Fuller M, et al. Differentiation of vascular smooth muscle cells from local precursors during embryonic and adult arteriogenesis requires notch signaling. Proc Natl Acad Sci U S A. 2012;109:6993-8. 
11. Gridley T. Notch signaling in the vasculature. Curr Top Dev Biol. 2010;92:277-309.

12. Rizzo P, Miele L, Ferrari R. The notch pathway: a crossroad between the life and death of the endothelium. Eur Heart J. 2013;34:2504-9.

13. Lassaletta AD, Chu LM, Robich MP, Elmadhun NY, Feng J, Burgess TA, et al. Overfed Ossabaw swine with early stage metabolic syndrome have normal coronary collateral development in response to chronic ischemia. Basic Res Cardiol. 2012;107:243.

14. Elmadhun NY, Lassaletta AD, Chu LM, Sellke FW. Metformin alters the insulin signaling pathway in ischemic cardiac tissue in a swine model of metabolic syndrome. J Thorac Cardiovasc Surg. 2013;145:258-65; discussion 265, 256.

15. Wang T, Baron M, Trump D. An overview of notch3 function in vascular smooth muscle cells. Prog Biophys Mol Biol. 2008:96:499-509.

16. Swift MR, Weinstein BM. Arterial-venous specification during development. Circ Res. 2009; 104:576-88.

17. Manderfield LJ, High FA, Engleka KA, Liu F, Li L, Rentschler S, et al. Notch activation of Jagged 1 contributes to the assembly of the arterial wall. Circulation. 2012; 125:314-23.

18. Chang AC, Patenaude A, Lu K, Fuller M, Ly M, Kyle A, et al. Notch-dependent regulation of the ischemic vasodilatory response-brief report. Arterioscler Thromb Vasc Biol. 2013;33:510-2.

19. Chang AC, Fu Y, Garside VC, Niessen K, Chang L, Fuller M, et al. Notch initiates the endothelial-to-mesenchymal transition in the atrioventricular canal through autocrine activation of soluble guanylyl cyclase. Dev Cell. 2011;21:288-300.

20. Su H, Takagawa J, Huang Y, Arakawa-Hoyt J, Pons J, Grossman W, et al. Additive effect of AAV-mediated angiopoietin-1 and VEGF expression on the therapy of infarcted heart. Int J Cardiol. 2009;133:191-7.

21. Tao Z, Chen B, Tan X, Zhao Y, Wang L, Zhu T, et al. Coexpression of VEGF and angiopoietin-1 promotes angiogenesis and cardiomyocyte proliferation reduces apoptosis in porcine myocardial infarction (MI) heart. Proc Natl Acad Sci U S A. 2011;108:2064-9.

22. Robich MP, Osipov RM, Nezafat R, Feng J, Clements RT, Bianchi C, et al. Resveratrol improves myocardial perfusion in a swine model of hypercholesterolemia and chronic myocardial ischemia. Circulation. 2010;122:S142-9.

23. Boodhwani M, Mieno S, Voisine P, Feng J, Sodha N, Li J, et al. High-dose atorvastatin is associated with impaired myocardial angiogenesis in response to vascular endothelial growth factor in hypercholesterolemic swine. J Thorac Cardiovasc Surg. 2006;132:1299-306.

24. Trask AJ, Katz PS, Kelly AP, Galantowicz ML, Cismowski MJ, West TA, et al. Dynamic micro- and macrovascular remodeling in coronary circulation of obese Ossabaw pigs with metabolic syndrome. J Appl Physiol. 2012;113:1128-40.

25. Bratz IN, Dick GM, Tune JD, Edwards JM, Neeb ZP, Dincer UD, et al. Impaired capsaicin-induced relaxation of coronary arteries in a porcine model of the metabolic syndrome. Am J Physiol Heart Circ Physiol. 2008;294:H2489-96.

26. Borbouse L, Dick GM, Asano S, Bender SB, Dincer UD, Payne GA, et al. Impaired function of coronary $\mathrm{BK}(\mathrm{Ca})$ channels in metabolic syndrome. Am J Physiol Heart Circ Physiol. 2009;297:H1629-37.

27. Quillard T, Devalliere J, Coupel S, Charreau B. Inflammation dysregulates notch signaling in endothelial cells: implication of notch2 and notch4 to endothelial dysfunction. Biochem Pharmacol. 2010;80:2032-41.

28. MacKenzie F, Duriez P, Larrivee B, Chang L, Pollet I, Wong F, et al. Notch4-induced inhibition of endothelial sprouting requires the ankyrin repeats and involves signaling through RBP-Jkappa. Blood. 2004;104:1760-8.

29. Yu B, Song B. Notch 1 signalling inhibits cardiomyocyte apoptosis in ischaemic postconditioning. Heart Lung Circ. 2014;23:152-8.

30. Nair P, Somasundaram K, Krishna S. Activated notch1 inhibits p53-induced apoptosis and sustains transformation by human papillomavirus type 16 e 6 and e7 oncogenes through a PI3K-PKB/AKT-dependent pathway. J Virol. 2003;77: 7106-12.

31. Sade H, Krishna S, Sarin A. The anti-apoptotic effect of notch-1 requires p56 ${ }^{\text {lck }}$-dependent, Akt/PKB-mediated signaling in T cells. J Biol Chem. 2004; 279:2937-44

32. Ford ES, Giles WH, Mokdad AH. Increasing prevalence of the metabolic syndrome among U.S. adults. Diabetes Care. 2004;27:2444-9.

\section{Discussion}

Dr Marc Ruel (Ottawa, Ontario, Canada). I wish to congratulate Dr Elmadhun and her team, in the laboratory of
Dr Frank Sellke, for an excellent presentation and for providing me with the report in advance for review.

The findings depicted this morning are important because they continue to increase our mechanistic understanding of the angiogenic processes in pathologic cardiovascular states, which is critical if these therapies are going to hopefully one day work in the patients for whom they are destined.

In the study, the deleterious effects brought on by the experimental re-creation of MetS did not exceed the formidable ability of pediatric swine to normalize myocardial perfusion in response to ameroid constrictor insertion. However, the core of the study is what happens "subclinically." You found that a number of angiogenic proteins are downregulated by MetS in this model and that apoptosis is increased. Several of the changes seemed to occur in the perivascular area of the blood vessels, likely involving smooth muscle cells.

I have the following questions.

Despite no differences in microsphere myocardial perfusion, is it possible that the pigs in the MetS group might have been more ischemic? In other words, are there limitations to microsphere-measured perfusion that would have been highlighted with imaging or by measuring endothelial-dependent or even endothelial-independent responses, considering that the localization of the angiogenic downregulation changes seemed to occur in the smooth muscle cells. Could new vessels in the MetS group have been less mature, or leakier, because of the changes in the smooth muscle cells? Were the left circumflex territory data compared with a control, non-intervened left anterior descending or right coronary artery territory?

Dr Elmadhun. Thank you very much, Dr Ruel. To address your questions: we did perform right and left heart catheterizations at harvest, and we sent those results to an interventional cardiologist who was able to quantify the Thrombolysis In Myocardial Infarction blush score and the Rentrop score. We did not find any differences between the CTL group and the MetS group; thus, we do not believe, given that data and the perfusion data, that the MetS group was more ischemic.

In terms of the microsphere data limitations, this is the reference standard to determine perfusion. Other methods can be used to examine myocardial perfusion. We have previously done magnetic resonance imaging studies and microvessel reactivity analysis. However, at our institution, we are unable to do magnetic resonance imaging studies. However, we did perform microvessel reactivity studies, and we found increased relaxation in the MetS group. This finding might have been related to the "thrifty genotype" of the Ossabaw miniswine, which has allowed them to survive on Ossabaw Island in the harsh feast and famine ecology.

Related to vessel leakiness and possible, immature vessels in the MetS group, this is very likely. However, we only used 1 harvest point. It would be interesting in future studies to see whether, as these vessels matured, if any difference was found in the vessel count at a later point or a difference was found in myocardial perfusion at a later point. This is an early MetS model. Perhaps if we had extended this study for 9 months or 1 year or had performed our study in adult, sexually mature animals, we might have seen even more differences. 